\title{
BOUNDED ANALYTIC FUNCTIONS AND METRICS OF CONSTANT CURVATURE ON RIEMANN SURFACES
}

\author{
By AKIRA YAMADA
}

\section{Introduction.}

Let $\boldsymbol{B}(\Omega)$ be the set of bounded analytic functions $f: \Omega \rightarrow \Delta$, where $\Omega$ is a simply connected hyperbolic Riemann surface and $\Delta$ is the unit disc. Let $\boldsymbol{B}_{0}(\Omega)$ be the set of locally schlicht functions belonging to $\boldsymbol{B}(\Omega)$. The Poincare metric $\lambda_{\Omega}$ of the surface $\Omega$ has constant curvature $\equiv-4$. Explicitly, we have $\lambda_{\Delta}(z)=$ $|d z| /\left(1-|z|^{2}\right)$. It is well known that the pull-back $f^{*} \lambda_{\Delta}(z)=\left|f^{\prime}(z) \| d z\right| /(1-$ $|f(z)|^{2}$ ) of $\lambda_{\Delta}$ via $f \in \boldsymbol{B}_{0}(\Omega)$ (resp. $f \in B(\Omega)$ ) is a metric of constant curvature $\equiv-4$ regular (resp. with isolated singularities) on $\Omega$. The main result of this paper asserts that the converse of the above relation holds. For simplicity, we denote by $M(X)$ the set of $\mathcal{C}^{\infty}$ conformal metrics of constant curvature $\equiv-4$ on a Riemann surface $X$.

THEOREM 1. For all $\lambda \in M(\Omega)$, there exists an $f \in \boldsymbol{B}_{0}(\Omega)$ such that $\lambda=f^{*} \lambda_{\Delta}$. Moreover, $\lambda=g * \lambda_{\Delta}$ for $g \in \boldsymbol{B}_{0}(\Omega)$ if and only if $g$ is of the form $g=\gamma \circ f$ where $\gamma \in \mathrm{Möb}(\Delta)$, the set of Möbius transformations leaving $\Delta$ fixed.

THEOREM 2. Let $E$ be an arbitrary closed discrete subset of $\Omega$. If $\lambda \in$ $M(\Omega \backslash E)$ has non-positive integral indices at every point in $E$, then there exists an $f \in \boldsymbol{B}(\Omega)$ such that $\lambda=f^{*} \lambda_{\Delta}$ on $\Omega \backslash E$. Moreover, $\lambda=g^{*} \lambda_{\Delta}$ on $\Omega \backslash E$ if and only if $g$ is of the form $g=\gamma \circ f$ with $\gamma \in \operatorname{Möb}(\Delta)$.

Theorems 1 and 2 show that the set of metrics of constant negative curvature is in a one-to-one correspondence with the set of bounded analytic functions in $\Omega$ modulo $\operatorname{Möb}(\Delta)$. Theorem 2 is an improvement of Theorem 29.1 in Heins [2] and many of the results concerning metrics of constant curvature in [2] are easy consequence of Theorem 1. Also, Theorem 1 allows us to define the monodromy homomorphism $\chi$ of a metric of constant curvature. Theorem 6 answers the question when the image of the homomorphism $\chi$ acts discontinuously on $\Delta$. In the last section, we prove a theorem which shows that a theorem in [5] is false.

The author wishes to thank the referee for helpful comments and advice.

Received December 8, 1987 


\section{Proof of theorem 1 .}

The following Lemma is useful.

LEMMA 3. Let $f: N \rightarrow \Omega$ be holomorphic where $N$ is a connected neighborhood of a simply connected hyperbolic Riemann surface $\Omega$. Assume that, for $z \in N, f$ satisfies the equality $f^{*} \lambda_{\Omega}(z)=\lambda_{\Omega}(z)$. Then $f$ is a restriction of a conformal automorphism of $\Omega$.

Proof. By conformal invariance, we may assume that $\Omega$ is the unit disc. But in this case Lemma 3 is well known. See [2, p. 39].

LEMMA 4. Let $X$ and $Y$ be surfaces of constant curvature $\kappa$. Then the surfaces $X$ and $Y$ are locally isometric.

Proof. This is well known. See [3, p. 169].

Proof of theorem 1. By conformal invariance, we may assume $\Omega=\Delta$. Applying Lemma 4 to the unit discs equipped with the metric $\lambda \in M(\Delta)$ and the Poincaré metric $\lambda_{\Delta}$, we have an open covering $\left\{U_{\alpha}\right\}_{\alpha \in \Delta}$ and a set of conformal (or anti-conformal) mappings $f_{\alpha}: U_{\alpha} \rightarrow \Delta(\alpha \in \Delta)$ with $\lambda \mid U_{\alpha}=f_{\alpha}^{*} \lambda_{\Delta}$. By taking $\bar{f}_{\alpha}$, if $f_{\alpha}$ is anti-conformal, we may assume that each $f_{\alpha}$ is conformal. Lemma 3 implies that there exists a set $\left\{\gamma_{\alpha \beta}\right\} \in \operatorname{Möb}(\Delta)$ such that $f_{\alpha}(z)=\gamma_{\alpha \beta}{ }^{\circ} f_{\beta}(z)$ for $z \in U_{\alpha} \cap U_{\beta}, \alpha, \beta \in \Delta$. Thus $f_{\alpha}(z)$ can be analytically continued along all paths contained in $\Delta$. Since $\Delta$ is simply connected, the monodromy theorem implies that there exists an $f \in \boldsymbol{B}_{0}(\Omega)$ satisfying $\lambda=f^{*} \lambda_{\Delta}$. The latter half of Theorem 1 is clear from Lemma 3 . This completes the proof.

\section{Monodromy homomorphisms inducd by metrics.}

Let $X$ be a hyperbolic Riemann surface. The Poincaré metric $\lambda_{X}$ on $X$ is defined by requiring the identity

$$
\lambda_{\Delta}=\pi * \lambda_{X}
$$

where $\pi: \Delta \rightarrow X$ is a holomorphic universal covering. Conversely, we note that if $\lambda \in M(X)$ satisfies the identity $\lambda_{\Delta}=\pi^{*} \lambda$ with $\pi: \Delta \rightarrow X$ holomorphic, then $\lambda$ is the Poincaré metric because we have $\lambda=\lambda_{X}$ from the inequality

$$
\lambda_{\Delta}=\pi * \lambda \leqq \pi * \lambda_{X} \leqq \lambda_{\Delta}
$$

This observation leads us to the following definition. Let $E$ be a (possibly empty) closed discrete subset of $X$. We call $\lambda \in M(X \backslash E)$ a branched Poincaré metric on $X$ with singularity on $E$ if $\lambda$ satisfies

$$
\lambda_{\Delta}=\pi * \lambda \quad \text { on } \quad \pi^{-1}(X \backslash E)
$$


for some holomorphic map $\pi: \Delta \rightarrow X$.

Lemma 5. Let $\lambda$ be a branched Poincaré metric on $X$ with singularity on $E$ satisfying $\lambda_{\Delta}=\pi * \lambda$. Then $\pi: \Delta \rightarrow X$ is a normal branched covering whose branch points are contained in E. (Here, we use the word "normal branched covering" to mean that the restriction $\pi \mid \pi^{-1}(X \backslash E)$ is a normal regular covering. [1, p. 38])

Conversely, every holomorphic normal branched covering $\pi: \Delta \rightarrow X$ yields a branched Poincaré metric $\lambda$ on $X$ with $\lambda_{\Delta}=\pi * \lambda$.

Proof. First, assume that $\lambda$ is a branched Poincaré metric. Let $G$ be the group $\{\gamma \in \operatorname{Möb}(\Delta) \mid \pi \circ \gamma=\pi$ on $\Delta\}$. It is clear that $G$ is discontinuous on $\Delta$ since $\pi$ is non-constant. We claim that $\pi(x)=\pi(y)$ for $x, y \in \Delta$ if and only if there exists a $\gamma \in G$ with $y=\gamma(x)$. Observe that by (3.1) the orders of the derivative $\pi^{\prime}$ at $x$ and $y$ are the same. Hence we can solve, at least locally, the equation $\pi \circ \gamma=\pi$ for $\gamma$ where $\gamma$ is holomorphic near $x$ and satisfies $y=\gamma(x)$. Thus,

$$
\lambda_{\Delta}(z)=\pi * \lambda(z)=(\pi \circ \gamma) * \lambda(z)=\gamma^{*} \pi * \lambda(z)=\gamma * \lambda_{\Delta}(z)
$$

for $z$ in a neighborhood of $x$. Then it follows from Lemma 3 that $\gamma$ is a restriction of a Möbius transformation fixing $\Delta$. By analytic continuation we conclude that $\gamma \in G$, proving the claim.

We next show that $\pi(\Delta)$ contains $X \backslash E$. Otherwise, it follows from the theory of S-K metrics due to Heins [2] that the upper envelope $\eta$ of the Perron family of metrics on $\pi(\Delta) \backslash E$ generated by $\lambda \mid \pi(\Delta) \backslash E$ and the Poincaré metric on $\pi(\Delta)$ belongs to the set $M(\pi(\Delta) \backslash E)$. $\eta$ satisfies the conditions $\eta>\lambda$ and $\pi^{*} \eta$ $\in M(\Delta)$, so that we have $\lambda_{\Delta}<\pi^{*} \eta$. This contradicts the maximality of the Poincaré metric $\lambda_{\Delta}$ in $M(\Delta)$. Thus $\pi(\Delta) \backslash E=X \backslash E$. Since the map $\pi$ is locally schlicht except on $\pi^{-1}(E)$, it is seen that the set of elliptic fixed points of $G$ is contained in $\pi^{-1}(E)$. We conclude that $X \backslash E$ is conformally equivalent to the quotient Riemann surface $\left(\Delta \backslash \pi^{-1}(E)\right) / G$ and that the map $\pi$ may be identified with the natural projection $\Delta \rightarrow \Delta / G$. Hence $\pi$ is a normal branched covering with possible branch points or punctures in $E$.

The second statement of the Lemma is proved similarly as in the case of regular coverings, and we omit its proof.

Now assume that $\pi: \Delta \rightarrow X$ is a holomorphic universal covering. Since $\pi * \lambda$ $\in M(\Delta)$ whenever $\lambda \in M(X)$, Theorem 1 guarantees the existence of an $f \in \boldsymbol{B}_{0}(\Delta)$ such that $\pi^{*} \lambda=f * \lambda_{\Delta}$. Noting that $\pi^{*} \lambda$ is $\Gamma$-invariant where $\Gamma$ is the covering group for the covering $\pi$, we conclude from Lemma 3 that there exists a homomorphism $\chi: \Gamma \rightarrow \operatorname{Möb}(\Delta)$ such that

$$
f \circ \gamma=\chi(\gamma) \circ f \quad \text { for all } \gamma \in \Gamma \text {. }
$$

We shall call the homomorphism $\chi$ the monodromy homomorphism induced by the metric $\lambda \in M(X)$. Observe that, for fixed $\pi, \chi$ is uniquely determined up to an inner automorphism of $\operatorname{Möb}(\Delta)$. It is natural to ask when is the image $\chi(\Gamma)$ discontinuous. The answer is given by the following: 
THEOREM 6. Let $\chi: \Gamma \rightarrow \operatorname{Möb}(\Delta)$ be the monodromy homomorphism induced by the metric $\lambda \in M(X)$. Then $\chi(\Gamma)$ acts discontinuously on $\Delta$ if and only if there exists a Riemann surface $Y$ and a branched Poincare metric $\eta$ on $Y$ such that $\lambda$ $=F^{*} \eta$ for some $F: X \rightarrow Y$ holomorphic.

Proof. Fix an $f \in \boldsymbol{B}_{0}(\Delta)$ such that $\pi^{*} \lambda=f^{*} \lambda_{\Delta}$ as above. First, assume that the group $\chi(\Gamma)$ is discontinuous on $\Delta$. Let $Y$ be the quotient Riemann surface $\Delta / \chi(\Gamma)$. Then by (3.2) $f$ induces a holomorphic map $F: X \rightarrow Y$ such that $F \circ \pi=$ $\pi_{1} \circ f$ where $\pi_{1}: \Delta \rightarrow Y$ denotes the natural projection. Let $\eta$ be the branched Poincaré metric on $Y$ determined by $\lambda_{\Delta}=\pi_{1}^{*} \eta$. Now we have

$$
\pi^{*} \lambda=f^{*} \lambda_{\Delta}=f^{*}\left(\pi_{1}^{*} \eta\right)=(F \circ \pi)^{*} \eta=\pi^{*}\left(F^{*} \eta\right),
$$

concluding that $\lambda=F^{*} \eta$, as desired.

Conversely, assume that $\lambda=F^{*} \eta$ where $F: X \rightarrow Y$ is holomorphic and $\eta$ is a branched Poincaré metric on $Y$. By Lemma 5 there exists a holomorphic normal branched covering $\pi_{1}: \Delta \rightarrow Y$ with $\lambda_{\Delta}=\pi_{1}^{*} \eta$. Let $E \subset Y$ be the set of singularities of $\eta$. Since both metrics $F^{*} \eta$ and $\pi_{1}^{*} \eta$ are regular, we find that $n(p, F)=n\left(q, \pi_{1}\right)$ for every pair $(p, q)$ such that $F(p)=\pi_{1}(q)$ where $n(p, F)$ denotes the multiplicity of $F$ at $p$. This observation allows us to conclude that there exists a holomorphic map $g: \Delta \rightarrow \Delta$ satisfying the condition $F \circ \pi=$ $\pi_{1} \circ g$. Thus we have

$$
\pi^{*} \lambda=\pi^{*}\left(F^{*} \eta\right)=\left(\pi_{1} \circ g\right)^{*} \eta=g^{*} \pi_{1}^{*} \eta=g^{*} \lambda_{\Delta},
$$

so that $f^{*} \lambda_{\Delta}=g^{*} \lambda_{\Delta}$. Lemma 3 shows that there exists a $r \in \operatorname{Möb}(\Delta)$ with $f=$ $\gamma \circ g$. Hence we conclude that $\chi(\Gamma) \subset \gamma \circ \Gamma_{1} \circ \gamma^{-1}$ where $\Gamma_{1}$ denotes the covering group of the branched covering $\pi_{1}$. This completes the proof of Theorem 6 since $\Gamma_{1}$ is discontinuous.

\section{Behavior of the metric at isolated singularities.}

As an application of Theorem 1 we study the behavior of a metric $\lambda$ of constant curvature arround a puncture. The problem being local, we may assume without loss of generality that $\lambda \in M(\Delta \backslash\{0\})$. Let $U$ be the upper halfplane. Since $\left(e^{\imath z}\right)^{*} \lambda \in M(U)$, by using Theorem 1 we obtain an $f \in \boldsymbol{B}_{0}(U)$ such that $\left(e^{i z}\right)^{*} \lambda=f^{*} \lambda_{\Delta}$. Let $\chi$ be the monodromy homomorphism induced by $\lambda$ and set $\gamma=\chi(\tau)$ where $\tau(z)=z+2 \pi$ is a generator for the covering group of $e^{\imath z}: U \rightarrow$ $\Delta \backslash\{0\}$. Hence we have that $f \circ \tau=\gamma \circ f$.

LEMMA 7. $\gamma$ is not a hyperbolic transformation.

Proof. Schwarz' lemma implies that $f$ is hyperbolically distance-decreasing. Thus,

$$
d_{\Delta}(f(z), \gamma(f(z)))=d_{\Delta}(f(z), f(\tau(z))) \leqq d_{U}(z, z+2 \pi),
$$


where $d_{\Delta}(\cdot, \cdot)$ and $d_{U}(\cdot, \cdot)$ denote the hyperbolic distance of $\Delta$ and $U$ respectively. Letting $z=i y \rightarrow \infty\left(y \in \boldsymbol{R}^{+}\right)$, we have

$$
\inf _{z \in \Delta} d_{\Delta}(z, \gamma(z))=0 \text {. }
$$

From this, it is easy to see that $\gamma$ is not hyperbolic.

LEMMA 8. The following estimates hold near the origin.

(i) If $\gamma$ is parabolic, then

$$
\lambda(z)=\frac{1}{2|z| \ln \left(C_{1} /|z|\right)}+O(1)
$$

with some constant $C_{1}>0$.

(ii) If $\gamma$ is elliptic, then there exist constants $C_{2}>0, C_{3}>0$ and an integer $k \geqq 0$ such that

$$
\lambda(z)=|z|^{\alpha+k-1}\left(C_{2}+O\left(z^{C_{3}}\right)\right),
$$

where $2 \pi \alpha(0<\alpha<1)$ is the rotation angle of $\gamma$ at a fixed point.

(iii) If $\gamma$ is the identity, then there exist $a$ constant $C_{4}>0$ and an integer $k \geqq 0$ such that

$$
\lambda(z)=|z|^{k}\left(C_{4}+O(z)\right) .
$$

Proof. (i) Theorem 1 implies that there exists a locally schlicht function $f: U \rightarrow U$ such that $\left(e^{\imath z}\right)^{*} \lambda=f^{*} \lambda_{U}$ and $f(z+2 \pi)=f(z)+2 \pi$ for all $z \in U$. Thus we have a Fourier series expansion

$$
f(z)=z+\sum a_{n} e^{n \imath z} .
$$

Since $\operatorname{Im} f(z)$ is periodic, it can be regarded as a positive harmonic function on $\Delta \backslash\{0\}$. Hence, substituting $w=e^{\imath z}$ we have

$$
\ln \frac{1}{|w|}+\operatorname{Im} \Sigma a_{n} w^{n}>0 \quad \text { for } \quad 0<|w|<1 .
$$

This inequality easily implies that $a_{n}=0$ for all $n<0$. Therefore,

$$
\lambda(w)|w|=\frac{\left|1+\sum_{n=0}^{\infty} n i a_{n} w^{n}\right|}{2 \ln (1 /|w|)+2 \operatorname{Im} \sum_{n=0}^{\infty} a_{n} w^{n}}=\frac{1}{2 \ln \left(C_{1} /|w|\right)}+O(w) \quad(w \rightarrow 0)
$$

with $C_{1}=e^{\operatorname{Im} a_{0}}$. This proves Case (i) of the Lemma.

(ii) As in Case (i) there exists a holomorphic function $f: U \rightarrow \Delta$ such that $\left(e^{\imath z}\right)^{*} \lambda=f^{*} \lambda_{\Delta}$ and that $f(z+2 \pi)=e^{2 \pi \alpha \imath} f(z), 0<\alpha<1$. Expanding $f$ in a Fourier series, we have

$$
f(z)=e^{\imath \alpha z} \sum_{n=k}^{\infty} a_{n} e^{n \imath z}
$$

where $k(\geqq 0)$ is an integer and $a_{k} \neq 0$. A similar calculation as in Case (i) yields the desired estimate. 
(iii) Since $\gamma$ is the identity, $f: U \rightarrow \Delta$ is of the form

$$
f(z)=\sum_{n=k}^{\infty} a_{n} e^{\imath n z}, \quad a_{k} \neq 0
$$

with an integer $k \geqq 0$. This suffices to conclude the proof of Lemma 8 .

We recall that the index $v$ at an isolated singularity $p$ of a metric of constant curvature $\lambda$ is defined by

$$
v=\lim _{z \rightarrow 0} \frac{\ln \lambda(z)}{\ln (1 /|z|)}
$$

where $z$ is a coordinate centered at $p$ (c.f. [2]). According to Lemma 8, this definition is legitimate and does not depend on the choice of the coordinate. We remark that the index $v$ satisfies the inequality $v \leqq 1$ and that the equality occurs if and only if the Möbius transformation $\gamma$ associated to the metric is parabolic. Also, observe that the index $v$ is a non-positive integer if and only if the associated transformation $\gamma$ is the identity.

In the proof of the last section we use the following corollary to Lemma 8 .

COROLLARY 1. ([4], p. 73) Let $\Omega$ be a hyperbolic plane region containing the origin. If $\lambda$ is the Poincaré metric on $\Omega \backslash\{0\}$, then we have

$$
\lim _{z \rightarrow 0} \lambda(z)|z| \ln (1 /|z|)=\frac{1}{2} .
$$

Proof. We may assume without loss of generality that $\Delta \backslash\{0\} \subset \Omega \backslash\{0\}$. Let $\pi_{1}\left(=e^{\imath z}\right): U \rightarrow \Delta \backslash\{0\}$ and $\pi: U \rightarrow \Omega \backslash\{0\}$ be holomorphic universal coverings. Let $f: U \rightarrow U$ be a lift of the inclusion map $\Delta \backslash\{0\} \rightarrow \Omega \backslash\{0\}$ with respect to the coverings $\pi_{1}$ and $\pi$. From the identities $\pi_{1}=\pi \circ f$ and $\pi * \lambda=\lambda_{\Delta}$, we have $f^{*} \lambda_{\Delta}=$ $\pi_{1}^{*} \lambda$. Since $f$ is a lift, there exists a $\gamma \in \Gamma$ such that $f(z+2 \pi)=\gamma \circ f(z)$, where $\Gamma$ is a cover transformation group for the covering $\pi$. Since $\Gamma$ does not contain elliptic transformations, Lemma 7 shows that $\gamma$ is either a parabolic or the identity element. By Lemma 8 it suffices to show that $\gamma$ is parabolic. Assume that $\gamma$ is the identity. Then there exists a holomorphic function $h: \Delta \backslash\{0\} \rightarrow U$ such that

$$
\pi \circ h=i d \text { on } \Delta \backslash\{0\} .
$$

By Riemann's removable singularity theorem, $h$ is extended to a holomorphic function on $\Delta$. This would contradicts the identity (4.1), since $0 \notin \pi(U)$. Hence $\gamma$ is parabolic, as desired.

\section{Proof of Theorem 2 .}

We consider the monodromy homomorphism $\chi: \Gamma \rightarrow \operatorname{Möb}(\Delta)$ induced by $\lambda \in$ $M(\Omega \backslash E)$ where $\Gamma$ is the covering group for the holomorphic universal covering $\pi: \Delta \rightarrow \Omega \backslash E$. By assumption every index $v$ is an integer $\leqq 0$. By similar 
reasoning as in the previous section concerning the classification of isolated singularities, we conclude that $\chi(\gamma)$ is the identity whenever $\gamma \in \Gamma$ is parabolic. Note that parabolic elements in $\Gamma$ correspond to the punctures of the region $\Omega \backslash E$. Since $\Omega$ is simply connected, we see that $\Gamma$ is generated by the parabolic elements, so that $\chi(\gamma)$ is the identity for all $\gamma \in \Gamma$. Hence $F$ is invariant under the covering group $\Gamma$ where $F$ is a function in $\boldsymbol{B}_{0}(\Delta)$ such that $\pi^{*} \lambda=F^{*} \lambda_{\Delta}$. We claim that $f=F \circ \pi^{-1}: \Omega \backslash E \rightarrow \Delta$ is the desired function. It is easy to see that $f$ is well defined and satisfies $\lambda=f^{*} \lambda_{\Delta}$. On the other hand, since $f$ is bounded, $f$ can be extended to a function in $\boldsymbol{B}(\Omega)$. This completes the proof.

\section{An inequality.}

Let $d(z)$ denote the maximal Euclidean radius of the schlicht discs centered at $f(z)$ which is contained in the Riemannian image of a regular function $f$. In 1970 Pommerenke proved in [5] the following inequality.

(*) There exists a constant $\beta<1$ such that

$$
\left(1-|z|^{2}\right)\left|f^{\prime}(z)\right| \leqq 2 \beta d(z)\left(1+\ln \frac{1}{d(z)}\right), \quad z \in \Delta,
$$

for every locally schlicht function $f: \Delta \rightarrow C$ with $\sup _{z \in \Delta} d(z) \leqq 1$.

Now we show, however, that such a constant $\beta<1$ does not exist.

THEOREM 9. There exists a unversal covering $f$ which does not satısfy the inequality $(*)$ for every $\beta<1$.

Proof. Let $E$ be the set $\{z \in \boldsymbol{C} \mid z=m+n, m, n \in \boldsymbol{Z}\}$ and consider a holomorphic universal covering $f: \Delta \rightarrow C \backslash E$. Then $f$ is locally schlicht and satisfies $\sup _{z \in \Delta} d(z) \leqq 1$. Let $\lambda(z)|d z|$ be the Poincaré metric on the domain $C \backslash E$. By definition, we have

$$
\lambda(f(z))\left|f^{\prime}(z)\right|=\frac{1}{1-|z|^{2}} .
$$

It is clear that $d(z)=|f(z)|$ if $|f(z)| \leqq 1 / 2$. Thus, for $|f(z)|$ small,

$$
\frac{\left(1-|z|^{2}\right) / f^{\prime}(z) \mid}{2 d(z)(1+\ln (1 / d(z)))}=\frac{1}{2 \lambda(w)|w| \ln (e /|w|)}
$$

with $w=f(z)$. By Corollary 1 , the right side of the above equation tends to 1 as $w=f(z) \rightarrow 0$. Hence the constant $\beta$ must satisfy $\beta \geqq 1$ as desired.

\section{REFERENCES}

[1] L. V. Ahlfors and L. Sario, Rıemann Surfaces, Princeton Univ. Press, Princeton, New Jersey, 1960. 
[2] M. Heins, On a class of conformal metrics, Nagoya Math. J. 21 (1962), 1-60.

[3] N. J. Hicks, Notes on Differential Geometry, Van Nostrand Reinhold, London, 1971.

[4] I. KRA, Automorphic Forms and Kleinian Groups, Benjamin, Reading, Massachusetts, 1972.

[5] С . Pommerenke, On Bloch functions, J. London Math. Soc. (2) 2 (1970), 689695.

Department of Mathematics

TOKyo GaKugei UNIVERsity

Nukuikita-Machi, Koganei-SHi

TOKYO 184, JAPAN 